Pacific Journal of Mathematics

AN ALGEBRAIC CLOSED GRAPH THEOREM 


\title{
AN ALGEBRAIC CLOSED GRAPH THEOREM
}

\author{
GABRIEL M. M. OBI
}

In this work we consider the question as to when an everywhere defined closed linear map from a quadratic space $H_{1}$ into another such space $H_{2}$ is orthocontinuous. The following result is proved:

Let $\left(H_{1}, \Phi_{1}\right),\left(H_{2}, \Phi_{2}\right)$ be quadratic spaces whose $\perp$-closed subspaces are semi-simple. If $T$ is an everywhere defined closed linear map on $H_{1}$ into $H_{2}$ then $T$ is orthocontinuous.

1. Introduction. In [1], [2] Piziak generalized, algebraically, the geometry of Hilbert space. He introduced the notion of quadratic space and with this studied sesquilinear forms in infinite dimensions. He showed that certain general results which are of pure algebra imply standard topological results in the context of Hilbert space (e.g., an analogue of the Riesz representation theorem was proved for these spaces and this implies the Riesz representation theorem for Hilbert spaces).

Now, in Hilbert space an everywhere defined linear operator is continuous iff its graph is closed. It is known that if $T$ is an everywhere defined linear operator on a quadratic space and if $T$ is orthocontinuous then the graph of $T$ is $\perp$-closed. The question is whether or not the converse of this is true. In [2] it is conjectured that this may not be true in general but that it may be true if our quadratic space is such that every $\perp$-closed subspace is splitting. In this work we show that this conjecture is true. In fact we show that if every $\perp$-closed subspace of our quadratic space is semi-simple then $T$ is orthocontinuous. We also consider other cases where $T$ is orthocontinuous but where $H_{1}, H_{2}$ are neither both anisotropic nor are both such that their $\perp$-closed subspaces are semi-simple.

One of the implications of our results is that in the case of inner-product spaces the completeness of the spaces is not necessary for the "algebraic closed graph theorem" to hold. Thus the theorem holds for pre-Hilbert spaces. Surprising as this may seem at first, we point out that the algebraic closed graph theorem does not imply the closed graph theorem. This is because there may be no context in a quadratic space in which to discuss continuity. Even if such a context exists it is possible for an orthocontinuous map not to be continuous as Example 4 shows. 


\section{Preliminaries.}

Definition 2.1. [2] A quadratic space is a triple $(K, H, \Phi)$ where $K$ is a division ring with involution $*$, say, $H$ is a left vector space over $K$ and $\Phi$ is a nondegenerate orthosymmetric sesquilinear form on $H$ with respect to the involutive anti-automorphism $*$ of $K$.

Definition 2.2. [2] Let $(K, H, \Phi)$ be a quadratic space. For $x, y$ in $H$ we say $x$ is orthogonal to $y$ and write $x \perp y$ iff $\Phi(x, y)=0$.

We note that since $\Phi$ is orthosymmetric, $x \perp y$ implies $y \perp x$ and conversely.

We shall in what follows suppress $K, \Phi$ if there is no danger of confusion and refer to a quadratic space $(K, H, \Phi)$ as $H$.

DEFINITION 2.3. [2] Let $H$ be a quadratic space. An element $x$ in $H$ is said to be isotropic iff $x \perp x$ and anisotropic otherwise. If every element in $H$ is anisotropic we say $H$ is anisotropic.

For any subset $A$ of $H$ put

$$
A^{\perp}=\{y \in H: y \perp x \text { for all } x \in A\} \text {. }
$$

It is easy to see that $A^{\perp}$ is a subspace of $H$ for every subset $A$ of $H$.

Definition 2.4. [1] A subspace $M$ of $H$ is said to be $\perp$-closed iff $M^{\perp \perp}=M$. If $H=M \oplus M^{\perp}$ we say that $M$ is a splitting subspace, and if $M \cap M^{\perp}=(0)$ we say that $M$ is semi-simple.

We point out that while it is true that every splitting subspace is $\perp$-closed and semi-simple it is not true in general that every semi-simple 'subspace is splitting. In fact a $\perp$-closed semi-simple subspace of a quadratic space $H$ may not split $H$, e.g., [1, Proposition 3.2.23]. If however $H$ is finite dimensional then every semisimple subspace is splitting [1, Corollary 3.5.8].

As pointed out in [2] the nature of the scalars and the possibility of existence of nonzero isotropic vectors are two main differences between Hilbert spaces and general quadratic spaces. (Isotropic vectors play an important role in some physical theories, e.g., in the geometry space-time with the Minkowskii metric [2].)

DEFINITION 2.5. Let $\left(H_{1}, \Phi_{1}\right),\left(H_{2}, \Phi_{2}\right)$ be quadratic spaces. Let $T: H_{1} \rightarrow H_{2}$ be a linear map. $T$ is said to be orthocontinuous if

$$
T\left(M^{\perp \perp}\right) \subseteq T(M)^{\perp \perp}
$$

for every subspace $M$ of $H_{1}$. 
Proposition 2.6. [2] Let $\left(H_{1}, \Phi_{1}\right),\left(H_{2}, \Phi_{2}\right)$ be quadratic spaces and $T: H_{1} \rightarrow H_{2}$ be a linear map. Then the following are equivalent:

(i) $M=M^{\perp \perp} \Longrightarrow T^{-1}(M)=T^{-1}(M)^{\perp \perp}$ for all subspaces $M$ of $H_{2}$.

(ii) If $M$ is a $\perp$-closed subspace of $H_{2}$ then $T^{-1}(M)$ is a $\perp$ closed subspace of $H_{1}$.

(iii) $T\left(M^{\perp \perp}\right) \subseteq T(M)^{\perp \perp}$ for all subspaces $M$ of $H_{1}$.

(iv) $\left(T^{-1}(M)\right)^{\perp \perp} \cong T^{-1}\left(M^{\perp \perp}\right)$ for all subspaces $M$ of $H_{2}$.

(v) $T$ is orthocontinuous.

In Hilbert space the restriction of a continuous map to a closed subspace is continuous. This is not the case in general in a quadratic space.

Proposition 2.7. Let $\left(H_{1}, \Phi_{1}\right),\left(H_{2}, \Phi_{2}\right)$ be quadratic spaces and $T: H_{1} \rightarrow H_{2}$ an orthocontinuous linear map. If $M$ is a $\perp$-closed semi-simple subspace of $H_{1}$ and $\Phi_{M}$ is the restriction of $\Phi_{1}$ to $M$ then $\left(K, M, \Phi_{M}\right)$ is a quadratic space. Further the restriction of $T$ to $M$ is orthocontinuous.

Proof. To show that $\left(M, \Phi_{M}\right)$ is a quadratic space it suffices to show that $\Phi_{M}$ is nondegenerate. Suppose $x \in M$ and $\Phi_{M}(x, y)=0$ for all $y \in M$. Then $x \in M^{\perp}$. But $M \cap M^{\perp}=(0)$; hence $x=0$. Hence $\Phi_{M}$ is nondegenerate.

Now let $A$ be a $\perp$-closed subspace of $H$ which is contained in $M$. We note that

$$
\left\{x \in M: \Phi_{M}(x, y)=0 \text { for all } y \in A\right\}=M \cap A^{\perp} .
$$

Thus the closure in $M$ of $A$ is $M \cap\left(M \cap A^{\perp}\right)^{\perp}$. But

$$
\begin{aligned}
M \cap\left(M \cap A^{\perp}\right)^{\perp} & =M \cap\left(M^{\perp} \vee A^{\perp \perp}\right) \\
& =M \cap M^{\perp} \vee M \cap A^{\perp \perp} \\
& =M \cap M^{\perp} \vee A \\
& =0 \vee A .
\end{aligned}
$$

i.e. Any $\perp$-closed subspace of $H$ contained in $M$ is $\perp$-closed in $M$. If $T_{M}$ is the restriction of $T$ to $M$ and $B$ is a $\perp$-closed subspace of $H_{2}$ then $T_{M}^{-1}(B)=M \cap T^{-1}(B)$. Since $M$ is $\perp$-closed in $H_{1}$ and $T$ is orthocontinuous we have that $T^{-1}(B) \cap M$ is a $\perp$-closed subspace of $H_{1}$ which is contained in $M$ and hence by the above argument is $\perp$-closed in $M$. Thus $T_{M}$ is orth ocontinuous.

3. Algebraic closed graph theorem.

DeFinition 3.1. Let $\left(H_{i}, \Phi_{i}\right) i=1,2$ be quadratic spaces and 
$T: H_{1} \rightarrow H_{2}$ be a linear map. The graph of $T$, written $G(T)$, is the set

$$
G(T)=\left\{(x, T x): x \in D_{T} \subseteq H_{1}\right\} .
$$

Proposition 3.2. Let $\left(H_{i}, \Phi_{i}\right) i=1,2$ be quadratic spaces. Then a subspace of $H_{1} \times H_{2}$ of the form $A \times B$ where $A$ and $B$ are subspaces of $H_{1}$ and $H_{2}$ respectively is $\perp$-closed in $H_{1} \times H_{2}$ iff $A$ is a $\perp$-closed subspace of $H_{1}$ and $B$ a $\perp$-closed subspace of $H_{2}$.

Proof. Let $(x, y) \in(A \times B)^{\perp}$. Then

$$
\begin{aligned}
0 & =\Phi_{1} \oplus \Phi_{2}((x, y),(u, v)) \quad \text { for all }(u, v) \in A \times B \\
& =\Phi_{1}(x, u)+\Phi_{2}(y, v) \quad \text { for all } \quad(u, v) \in A \times B .
\end{aligned}
$$

In particular for $u=0$ we have that $y \in B^{\perp} \subseteq H_{2}$. Similarly $x \in A^{\perp} \subseteq$ $H_{1}$. Hence $(x, y) \in A^{\perp} \times B^{\perp}$. It is clear that if $(x, y) \in A^{\perp} \times B^{\perp}$ then $(x, y) \in(A \times B)^{\perp}$. Thus $(A \times B)^{\perp}=A^{\perp} \times B^{\perp}$. If $A \times B$ is a $\perp$-closed subspace of $H_{1} \times H_{2}$ we have that

$$
\begin{aligned}
(A \times B) & =(A \times B)^{\perp \perp} \\
& =A^{\perp \perp} \times B^{\perp \perp} .
\end{aligned}
$$

So, $A=A^{\perp \perp}$ and $B=B^{\perp \perp}$. Conversely if $A^{\perp \perp}=A$ and $B^{\perp \perp}=B$ then from $(A \times B)^{\perp}=A^{\perp} \times B^{\perp}$ we have that $A \times B$ is a $\perp$-closed subspace of $H_{1} \times H_{2}$.

CoROLlary 3.3. Let $\left(H_{i}, \Phi_{i}\right) i=1,2$ be quadratic spaces and let $\pi: H_{1} \times H_{2} \rightarrow H_{1}$ (resp. $\pi_{2}: H_{1} \times H_{2} \rightarrow H_{2}$ ) be the linear map defined by

$$
\pi((x, y))=x\left(\operatorname{resp} . \pi_{2}((x, y))=y\right) \quad \text { for all }(x, y) \in H_{1} \times H_{2} .
$$

Then for any subspace $A$ of $H_{1}$ (resp. $B$ of $\left.H_{2}\right) \pi^{-1}(A)$ (resp. $\left.\pi_{2}^{-1}(B)\right)$ is $\perp$-closed in $H_{1} \times H_{2}$ iff $A$ is $\perp$-closed in $H_{1}$, (resp. iff $B$ is $\perp$-closed in $H_{2}$ ).

Proof. $\pi^{-1}(A)=A \times H_{2}$ for any subset $A$ of $H_{1}$. This by Proposition 3.2 we have that $A \times H_{2}$ is a $\perp$-closed subspace of $H_{1} \times H_{2}$ iff $A$ is a $\perp$-closed subspace of $H_{1}$. (The proof for $\pi_{2}$ proceeds similarly.)

Proposition 3.4. Let $M$ be a splitting subspace of a quadratic space $(H, \Phi)$. Then there exists a * sesquilinear form $\Psi$ on $H / M^{\perp}$ with respect to which $\left(H / M^{\perp}, \Psi\right)$ is a quadratic space. Moreover if $p$ is the canonical map $p: H \rightarrow H / M^{\perp}$ and if a subspace $A$ of $H / M^{\perp}$ is $\perp$-closed then $p^{-1}(A)$ is a $\perp$-closed subspace of $H$. If $H$ is of 
the form $\left(H_{1} \times H_{2}, \Phi_{1} \oplus \Phi_{2}\right)$ where $\left(H_{i}, \Phi_{i}\right) i=1$, 2 are quadratic spaces and $M=H_{1} \times(0)$, say, then a subspace $A$ of $H / M^{\perp}$ is $\perp$-closed iff $p^{-1}(A)$ is a 1 -closed subspace of $H$.

Proof. Since $M$ is a splitting subspace it is semi-simple and hence the restriction of $\Phi$ to $M$, $\Phi_{M}$, say is such that $\left(M, \Phi_{M}\right)$ is a quadratic space, by Proposition 2.7. Also since $M$ is a splitting subspace there exists a projection $P$ on $H$ such that $M=\operatorname{Im}(P)$ [1]. Thus $M^{\perp}=\operatorname{Ker} P$ and so there exists an isomorphism $\phi: H / M^{\perp} \rightarrow M$. For $[x],[y] \in H / M^{\perp}$ define

$$
\Psi([x],[y])=\Phi_{M}(\phi([x]), \phi([y])) .
$$

We then have that

$$
\begin{aligned}
& \Psi([x],[y])=0 \quad \text { for all }[x] \in H / M^{\perp} \\
& \Longleftrightarrow \Phi_{M}(\phi([x]), \phi([y]))=0 \quad \text { for all } \phi([x]) \in M \\
& \Longleftrightarrow \phi([y])=0 \quad \text { since } \Phi_{M} \text { is nondegenerate and } \phi \text { is onto } \\
& \Longleftrightarrow[y]=0 \quad \text { since } \phi \text { is } 1-1 .
\end{aligned}
$$

Therefore $\Psi$ is nondegenerate. It is easy to see that $\Psi$ is orthosymmetric and $*$ sesquilinear relative to the involutive anti-automorphism * of $K$. So, $\left(H / M^{\perp}, \Psi\right)$ is a quadratic space. Let $A \subseteq H / M^{\perp}$.

$$
\begin{aligned}
{[x] \in A^{\perp} } & \Longleftrightarrow \Phi_{M}(\phi([x]), \phi([y]))=0 \forall[y] \in A \\
& \Longleftrightarrow \phi([y]) \in \phi(A)^{\perp} .
\end{aligned}
$$

From this we obtain $\phi\left(A^{\perp}\right)=\phi(A)^{\perp}$ and hence that $\phi$ maps $\perp$-closed subspaces of $H / M^{\perp}$ into $\perp$-closed subspaces of $M$. Therefore $\phi^{-1}$ is orthocontinuous. Also if $B^{\perp \perp}=B$ in $M$, we have that there exists an $A \subseteq H / M^{\perp}$ such that $A^{\perp \perp}=A$ in $H / M^{\perp}$ and $\phi(A)=B$. Indeed, since $\phi$ is onto there exists $A \subseteq H / M^{\perp}$ such that $\phi(A)=B$.

$$
\begin{aligned}
B & =B^{\perp \perp} \\
& =\phi(A)^{\perp \perp} \\
& =\phi\left(A^{\perp \perp}\right) .
\end{aligned}
$$

Hence

$$
\begin{aligned}
A & =\phi^{-1}(B) \\
& =\phi^{-1}\left(\phi\left(A^{\perp \perp}\right)\right) \\
& =A^{\perp \perp}
\end{aligned}
$$

since $\phi$ is a bijection. Thus every $\perp$-closed subspace of $M$ is the image of a $\perp$-closed subspace of $H / M^{\perp}$, i.e., if $B$ is a $\perp$-closed subspace of $M$ then $\phi^{-1}(B)$ is a $\perp$-closed subspace of $H / M^{\perp}$. Hence $\varphi$ is orthocontinuous. Now consider the following diagram: 


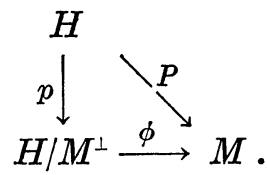

Suppose $A$ is a $\perp$-closed subspace of $H / M^{\perp}$. Since

$$
p^{-1}(A)=P^{-1}(\phi(A))
$$

and $P$ being a projection is orthocontinuous [1] we have that $p^{-1}(A)$ is a $\perp$-closed subspace of $H$. Finally suppose $H=\left(H_{1} \times H_{2}, \Phi_{1} \oplus \Phi_{2}\right)$ then $H_{1} \times\{0\},\{0\} \times H_{2}$ are $\perp$-closed subspaces of $H$ which split $H$. Put $M=H_{1} \times\{0\}$. By Corollary 3.3 we have that if $B$ is a subspace of $H_{1} \cong H_{1} \times\{0\}$ then $P^{-1}(B)$ is $\perp$-closed in $H=H_{1} \times H_{2}$ iff $B$ is $\perp$-closed in $H_{1}$. Therefore $p^{-1}(A)=P^{-1}(\phi(A))$ is $\perp$-closed in $H_{1} \times H_{2}$ iff $\phi(A)$ is $\perp$-closed in $M$ iff $A$ is $\perp$-closed in $H / M^{\perp}$.

CoROLlaRY 3.5. Let $\left(H_{i}, \Phi_{i}\right) i=1,2$ be quadratic spaces. Let $\pi: H_{1} \times H_{2} \rightarrow H_{1}$ be defined by $\pi((x, y))=x$ for all $(x, y)$ in $H_{1} \times H_{2}$. Then $\pi$ maps $\perp$-closed subspaces of $H_{1} \times H_{2}$ onto $\perp$-closed subspaces of $H_{1}$.

Proof. Consider the following diagram:

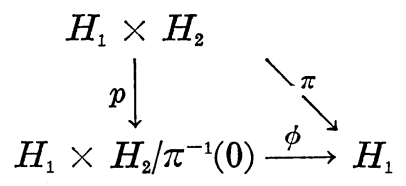

where $p$ and $\phi$ are the mappings defined in Proposition 3.4. Let $A$ be a $\perp$-closed subspace of $H_{1} \times H_{2}$. Then $A=p^{-1}(B)$ for some $B$ in $H_{1} \times H_{2} / \pi^{-1}(0)$. By Proposition 3.4 we have that $B$ is $\perp$-closed in $H_{1} \times H_{2} / \pi^{-1}(0)$. Thus

$$
\begin{aligned}
\pi(A) & =\phi(p(A)) \\
& =\phi\left(p\left(p^{-1}(B)\right)\right) \\
& =\phi(B)
\end{aligned}
$$

is $\perp$-closed in $H_{1}$.

REMARK. We note that if $M$ is a $\perp$-closed subspace of a quadratic space $H$ although it is not true in general that the intersection of a $\perp$-closed subspace of $H$ and $M$ is $\perp$-closed in $M$ it is however true that every $\perp$-closed subspace of $M$ is $\perp$-closed in $H$. Indeed if $A \subseteq M$ then the closure of $A$ in $M$ is $M \cap\left(M \cap A^{\perp}\right)^{\perp}$ which is a $\perp$-closed subspace of $H$. It follows therefore that if $T$ is a linear transformation on $H$ which maps $\perp$-closed subspaces into $\perp$-closed 
subspaces, the restriction of $T$ to any $\perp$-closed subspace, $M$, of $H$ maps $\perp$-closed subspaces of $M$ into $\perp$-closed subspaces of the co-domain of $T$.

THEOREM 3.6. Let $\left(H_{i}, \Phi_{i}\right) i=1,2$ be quadratic spaces such that every $\perp$-closed subspace of $H_{1}, H_{2}$ is semi-simple. Let $T: H_{1} \rightarrow H_{2}$ be an every where defined closed linear map. Then $T$ is orthocontinuous.

Proof. We first note that since every $\perp$-closed subspace of $H_{1}, H_{2}$ is semi-simple the same is true of every $\perp$-closed subspace of $H_{1} \times H_{2}$. Hence, since $G(T)$ is $\perp$-closed we have that $G(T) \cap$ $G(T)^{\perp}=(0)$ and that the restriction of any orthocontinuous linear map on $H_{1} \times H_{2}$ to $G(T)$ is orthocontinuous. Let $\pi_{1}: H_{1} \times H_{2} \rightarrow H_{1}$ be defined by $\pi_{1}(x, y)=x$ for every $(x, y)$ in $H_{1} \times H_{2}$. By Corollary $3.5 \pi_{1}$ maps $\perp$-closed subspaces onto $\perp$-closed subspaces. The restriction of $\pi_{1}$ to $G(T), \pi_{1 / G(T)}$, is 1-1, onto and by the Remark maps $\perp$ closed subspaces of $G(T)$ onto $\perp$-closed subspaces of $H_{1}$. Therefore $\pi_{1 / G(T)}^{-1}$ is orthocontinuous. Also $\pi_{2}: H_{1} \times H_{2} \rightarrow H_{2}$ defined by $\pi_{2}((x, y))=y$ for all $(x, y) \in H_{1} \times H_{2}$ is orthocontinuous by Corollary 3.3. Therefore its restriction to $G(T), \pi_{2 / G(T)}$, is orthocontinuous.

Now

$$
T x=\pi_{2 / G(T)} \circ \pi_{1 / G(T)}^{-1}(x)
$$

which is orthocontinuous.

An observation of the proof of the theorem shows that if the graph of $T$ is semi-simple then $T$ is orthocontinuous. We now consider other cases where the conditions on $H_{1}, H_{2}$ imply this and hence the orthocontinuity of $T$.

COROLlaRY 3.3. Let $\left(H_{i}, \Phi_{i}\right) i=1,2$ be anisotropic quadratic spaces. If $T$ is an everywhere defined closed linear map on $H_{1}$ into $\mathrm{H}_{2}$ then $\mathrm{T}$ is orthocontinuous.

Proof. Since $\left(H_{i}, \Phi_{i}\right) i=1,2$ is anisotropic so also is $\left(H_{1} \times H_{2}\right.$, $\left.\Phi_{1} \oplus \Phi_{2}\right)$ as can be easily checked. Hence every $\perp$-closed subspace of $H_{1} \times H_{2}$ is splitting. Since a splitting subspace is semi-simple we have that every $\perp$-closed subspace of $H_{1} \times H_{2}$ is semi-simple. The result then follows from the theorem.

REMARK. Corollary 3.7 establishes Piziak's conjecture [2].

Proposition 3.8. Let $\left(H_{i}, \Phi_{i}\right) i=1,2$ be quadratic spaces over a division ring $K$. Suppose there exists a subset $R$ of $K$ with the 
following properties:

(i) $0 \varepsilon \pm R$

(ii) $R \cap-R=\{0\}$.

If $\Phi_{1}(x, x), \Phi_{2}(y, y) \in R$ for all $x \in H_{1}, y \in H_{2}$ and if $H_{1}$ is anisotropic then an everywhere defined closed linear map $T: H_{1} \rightarrow H_{2}$ is orthocontinuous.

Proof. In view of the observation made after the proof of the theorem, it suffices to show that $G(T)$ is semi-simple. Suppose $(x, T x) \in G(T) \cap G(T)^{\perp}$ then

$$
\begin{aligned}
\Phi(x, x)+\Phi_{2}(T x, T x) & =\Phi_{1} \oplus \Phi_{2}((x, T x),(x, T x)) \\
& =0 . \\
\therefore \quad \Phi_{1}(x, x) & =-\Phi_{2}(T x, T x) .
\end{aligned}
$$

But $\Phi_{1}(x, x) \in R$ and $\Phi_{2}(T x, T x) \in R$. Thus if $(x, T x) \in G(T) \cap G(T)^{\perp}$ we have that $\Phi_{1}(x, x) \in R \cap-R$. But $R \cap-R=\{0\}$. Therefore $\Phi_{1}(x, x)=0$. Since $H_{1}$ is anisotropic we have that $x=0$ and so $(x, T x)=(0,0)$ so $G(T)$ is semi-simple.

REMARK. (1) If in the above proposition $T$ is $1-1$ and $H_{2}$ is anisotropic while $H_{1}$ is allowed to be arbitrary the same result is obtained.

(2) As pointed out in the introduction, our results imply that in the case of inner product space the completeness of the spaces is not necessary for an everywhere defined closed linear map to be orthocontinuous. We pointed out also that even if there is a context in which to discuss continuity it is possible for an orthocontinuous map not to be continuous. We now give an example.

4. Example. Let $K=R^{1}, H_{1}=H_{2}=R^{4}$. Define $\Phi_{1}$ on $H_{1}$ by

$$
\Phi_{1}\left(\left(x_{1}, x_{2}, x_{3}, x_{4}\right),\left(y_{1}, y_{2}, y_{3}, y_{4}\right)\right)=\sum_{1}^{3} x_{i} y_{i}-x_{4} y_{4}
$$

and $\Phi_{2}$ on $H_{2}$ by

$$
\Phi_{2}\left(\left(x_{1}, x_{2}, x_{3}, x_{4}\right),\left(y_{1}, y_{2}, y_{3}, y_{4}\right)\right)=\sum_{1}^{4} x_{i} y_{i} .
$$

Then $\left(H_{i}, \Phi_{i}\right) i=1,2$ are quadratic spaces. In fact $H_{2}$ is a Hilbert space as is well known. Let $\mathscr{T}$ be a collection of subsets of $H_{1}$ consisting of $\varnothing, H_{1}$, and all subsets $A$ of $H_{1}$ for which there exists a $\perp$-closed subspace $M \subseteq H_{1}$ with $A \subseteq H_{1} \sim M$. Then

(i) $\varnothing, H_{1} \in \mathscr{T}$ by definition.

(ii) If $A_{\alpha} \in \mathscr{T}$ we have that $\mathrm{U}_{\alpha} A_{\alpha} \in \mathscr{T}$. 
Indeed since for each $\alpha$ there exists a $\perp$-closed subspace $M_{\alpha}$ such that $A_{\alpha} \subseteq H_{1} \sim M_{\alpha}$ we have $\mathrm{U}_{\alpha} A_{\alpha} \subseteq \mathrm{U}_{\alpha} H_{1} \sim M_{\alpha}=H_{1} \sim \bigcap_{\alpha} M_{\alpha}$. But $\bigcap_{\alpha} M_{\alpha}$ is $\perp$-closed. Therefore $\mathrm{U}_{\alpha} A_{\alpha} \in \mathscr{T}$.

(iii) If $A_{k} k=1,2, \cdots, n \in \mathscr{T}$ then $\bigcap_{k=1}^{n} A_{k} \in \mathscr{T}$. For $\bigcap_{k=1}^{n} A_{k} \subseteq A_{k}$ for all $k=1,2, \cdots, n$, and $A_{k} \leqq H_{1} \sim M_{k}$ for some $\perp$-closed subspace $M_{k}$ of $H_{1}$.

Therefore $\mathscr{T}$ is a topology for $H_{1}$. Let $\mathscr{C}$ be the usual topology of $R^{4}$ which as is known arises from $\Phi_{2}$ in a natural way. Now let $I:\left(H_{1}, \mathscr{T}\right) \rightarrow\left(H_{2}, \mathscr{C}\right)$ be the map defined by $I x=x$ for all $x \in H_{1}$. Then $I$ is orthocontinuous since the inverse image of a $\perp$-closed subspace of $\left(H_{2}, \Phi_{2}\right)$ is a finite dimensional subspace of $\left(H_{1}, \Phi_{1}\right)$ and hence by [1, Corollary 3.5.2] is a $\perp$-closed subspace of $\left(H_{1}, \Phi_{1}\right)$. But $I$ is not continuous for if it were $I^{-1}\left(\left\{x \in H_{2}:\|x\|<1\right\}\right)$ would be an open set in $\left(H_{1}, \mathscr{T}\right)$ and hence would not contain 0 . This is a contradiction since $0 \in I^{-1}\left(\left\{x \in H_{2}:\|x\|<1\right\}\right)$.

\section{REFERENCES}

1. R. Piziak, An Algebraic Generalization of Hilbert Space Geometry, Ph. D. Thesis University of Massachusetts 1969.

2. - Sesquilinear forms in infinite dimensions, Pacific J. Math., 43 (1972), $475-481$.

Received May 17, 1977.

UNIVERSITY OF BENIN

P. M. B. 1154

BENIN CITY, NigeRIA 



\section{PACIFIC JOURNAL OF MATHEMATICS}

\section{EDITORS}

RICHARD ARENS (Managing Editor)

University of California

Los Angeles, California 90024

C. W. Curtis

University of Oregon

Eugene, OR 97403

C. C. MOORE

University of California

Berkeley, CA 94720

\section{J. DUGUNDJI}

Department of Mathematics University of Southern California Los Angeles, California 90007

R. Finn AND J. Milgram Stanford University Stanford, California 94305

ASSOCIATE EDITORS
E. F. BECKENBACH

B. H. NeUMANN

F. WOLF

K. YoshidA

\section{SUPPORTING INSTITUTIONS}

UNIVERSITY OF BRITISH COLUMBIA UNIVERSITY OF SOUTHERN CALIFORNIA CALIFORNIA INSTITUTE OF TECHNOLOGY STANFORD UNIVERSITY UNIVERSITY OF CALIFORNIA MONTANA STATE UNIVERSITY UNIVERSITY OF TOKYO UNIVERSITY OF NEVADA, RENO UNIVERSITY OF UTAH NEW MEXICO STATE UNIVERSITY OREGON STATE UNIVERSITY UNIVERSITY OF OREGON

WASHINGTON STATE UNIVERSITY UNIVERSITY OF WASHINGTON OSAKA UNIVERSITY 


\section{Pacific Journal of Mathematics}

\section{Vol. 74, No. $1 \quad$ May, 1978}

Gerald Arthur Anderson, Computation of the surgery obstruction groups

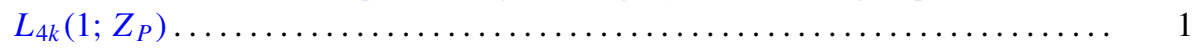

R. K. Beatson, The degree of monotone approximation ................ 5

Sterling K. Berberian, The character space of the algebra of regulated functions . . . 15

Douglas Michael Campbell and Jack Wayne Lamoreaux, Continua in the plane with

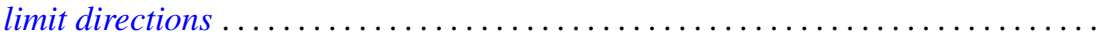

R. J. Duffin, Algorithms for localizing roots of a polynomial and the Pisot

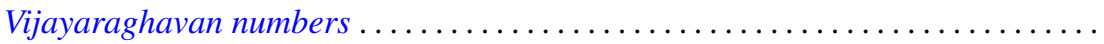

Alessandro Figà-Talamanca and Massimo A. Picardello, Functions that operate on

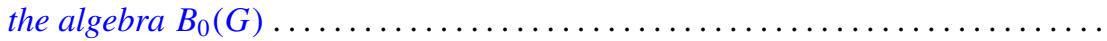

John Erik Fornaess, Biholomorphic mappings between weakly pseudoconvex

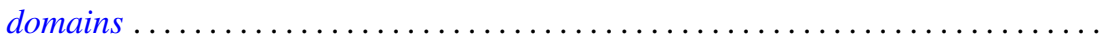

Andrzej Granas, Ronald Bernard Guenther and John Walter Lee, On a theorem of S.

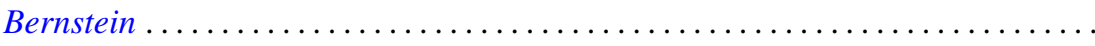

Jerry Grossman, On groups with specified lower central series quotients . .........

William H. Julian, Ray Mines, III and Fred Richman, Algebraic numbers, a constructive development . . . . . . . . . . . . . . . . . . . . . . .

Surjit Singh Khurana, A note on Radon-Nikodým theorem for finitely additive

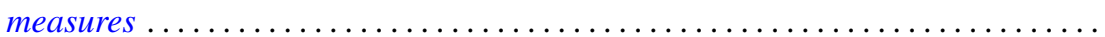

Garo K. Kiremidjian, A Nash-Moser-type implicit function theorem and nonlinear

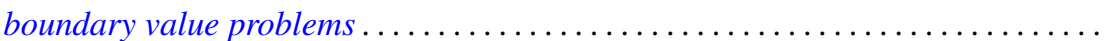

Ronald Jacob Leach, Coefficient estimates for certain multivalent functions ....

John Alan MacBain, Local and global bifurcation from normal eigenvalues. II . . 133

James A. MacDougall and Lowell G. Sweet, Three dimensional homogeneous algebras...

John Rowlay Martin, Fixed point sets of Peano continua ......

R. Daniel Mauldin, The boundedness of the Cantor-Bendixson order of some analytic sets...

Richard C. Metzler, Uniqueness of extensions of positive linear functions ..

Rodney V. Nillsen, Moment sequences obtained from restricted powers . .

Keiji Nishioka, Transcendental constants over the coefficient fields in differential elliptic function fields...

Gabriel Michael Miller Obi, An algebraic closed graph theorem

Richard Cranston Randell, Quotients of complete intersections by $\mathbf{C}^{*}$ actions . . 221

Bruce Reznick, Banach spaces which satisfy linear identities . .

Bennett Setzer, Elliptic curves over complex quadratic fields...

Arne Stray, A scheme for approximating bounded analytic functions on certain subsets of the unit disc.

Nicholas Th. Varopoulos, A remark on functions of bounded mean oscillation and bounded harmonic functions. Addendum to: "BMO functions and the

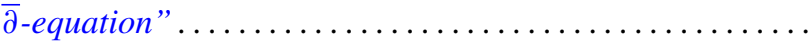

Charles Irvin Vinsonhaler, Torsion free abelian groups quasi-projective over their

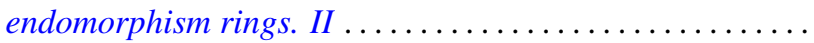

Thomas R. Wolf, Characters of $p^{\prime}$-degree in solvable groups ... 